\title{
Is it still relevant to consider polysomnography as essential for noninvasive ventilation titration?
}

\author{
Jean-Christian Borel (10 ${ }^{1,2}$ and Jesus Gonzalez-Bermejo ${ }^{3,4}$ \\ Affiliations: ${ }^{1}$ Département recherche et Développement, AGIR à dom, Meylan, France. ${ }^{2}$ Laboratoire HP2, \\ INSERM U 1042, Université Grenoble-Alpes, Grenoble, France. ${ }^{3}$ Sorbonne Université-médecine, INSERM, \\ UMRS1158 Neurophysiologie respiratoire expérimentale et clinique, Paris, France. ${ }^{4}$ AP-HP, Groupe \\ Hospitalier Pitié-Salpêtrière Charles Foix, Service de Pneumologie et Réanimation Médicale (Département \\ "R3S"), Paris, France.
}

Correspondence: Jean-Christian Borel, AGIR à dom Association, Research and Development Dpt, 36 Bd du Vieux Chêne, 38240 Meylan, France. E-mail: j.boreldagiradom.com

@ERSpublications

Titration of noninvasive ventilation by polysomnography reduces patient-ventilator asynchrony and may be useful to improve treatment tolerance in patients who are struggling with NIV http://bit.ly/2v1RDty

Cite this article as: Borel J-C, Gonzalez-Bermejo J. Is it still relevant to consider polysomnography as essential for noninvasive ventilation titration? Eur Respir J 2019; 53: 1900619 [https://doi.org/10.1183/ 13993003.00619-2019].

Setting the parameters of long-term nocturnal noninvasive ventilation (NIV) is crucial for effective correction of alveolar hypoventilation and respiratory events during sleep. Moreover, the treatment must be comfortable to ensure patient compliance from the beginning. Adaptation to NIV is usually carried out during daytime. The problem is that daytime behaviours may not reflect sleep ventilatory patterns and since respiratory events persist during sleep, mismatches between the patient and the ventilator may result [1]. It is widely believed that asynchrony events reduce the quality of ventilation and sleep, thus clinicians frequently attempt to identify the different types of asynchrony, their causes and how to limit their effects [2]. Titration of NIV by attended polysomnography (PSG) is considered to be the gold standard for the observation and correction of such events. However, PSG is complex, it may be difficult to access and the benefits of carrying out systematic PSG as part of a routine assessment remains in debate [3].

In this issue of the European Respiratory Journal, HanNAN et al. [4] have reported the results of the largest randomised controlled trial to date evaluating the benefits of titration of NIV by attended PSG. Using robust methodology, titration by attended PSG reduced patient-ventilator asynchrony in comparison with daytime, bedside titration. Nevertheless, sleep fragmentation (the co-primary outcome) was not reduced after 10 weeks of treatment. Moreover, the secondary outcomes (gas exchange, sleepiness, sleep quality, quality of life and adverse events of NIV) did not differ between the two modes of titration. Overall, the results confirmed findings suggested by a pilot study by PATOUT et al. [5] in patients with overlap syndrome.

In accordance with previous studies [1, 6, 7], HANNAN et al. [4] reported that the most frequent asynchrony event was ineffective respiratory efforts. In contrast, however, FANFulLa et al. [8] found that in a sample of patients with neuromuscular pathologies the reduction of ineffective efforts was achieved following optimisation of the NIV settings and that this reduction was also associated with an 
improvement in gas exchange and sleep structure. FANFulla et al. [8] used a spontaneous NIV mode (with no back-up respiratory rate) so that if patients did not trigger their ventilator, their ventilation was not pressure-supported. These results indicated that the impact of the asynchrony on ventilation effectiveness also depends on the interaction between the underlying pathology and the NIV settings. In the study of HANNAN et al. [4], the back-up rate was a mean \pm SD of $13.5 \pm 2$ cycles per minute; this may have limited the impact of the asynchrony on gas exchange. Furthermore, the back-up rate was not lowered in any of the patients who underwent titration by PSG, and was actually raised in $20 \%$ of the patients in that group.

Surprisingly, the mean level of pressure support (PS) used in the study of HanNan et al. [4] was quite low (mean \pm SD $6.7 \pm 2 \mathrm{cmH}_{2} \mathrm{O}$ ), which reflected the fact that the patients were stable and only in the first stages of respiratory insufficiency. The approach contrasts, however, with the "high-intensity ventilation" strategy that attempts to control nocturnal hypoventilation as much as possible [9], although studies that have demonstrated positive effects of this strategy have mostly evaluated patients with COPD [9]. The majority of the patients in the sample of HANNAN et al. [4] had neuromuscular diseases; nevertheless, the results showed that mean nocturnal $\mathrm{CO}_{2}$ pressure remained above $49-50 \mathrm{mmHg}$ for $25 \%$ of the patients, which suggests there could be a lack of control of ventilation. This is important because it could have a negative impact on the individual's prognosis in the long term [10]. It was not possible to determine from the results if the low-pressure strategy (both PS and expiratory positive airway pressure) contributed to the asynchrony; in the group titrated with PSG, two thirds of the patients required adaptation of the expiratory positive airway pressure $\left(\right.$ mean $\left.+1.4 \mathrm{cmH}_{2} \mathrm{O}\right)$ and half required adaptation of the $\mathrm{PS}\left(\right.$ mean $+0.6 \mathrm{cmH}_{2} \mathrm{O}$ ).

Another important finding of the study by HANNAN et al. [4] was that after titration with PSG, adherence to NIV improved in the subgroup of patients who previously had shown poor treatment compliance $(<4 \mathrm{~h}$ per night) during the period of acclimatisation (2-3 weeks). A similar improvement was not noted in the control group, who only underwent daytime clinical titration. Although exploratory, this finding supports the assumption that titration by PSG could improve both treatment adherence and quality of ventilation in patients with low tolerance after clinical titration using simple monitoring tools [11]. These findings are in line with the results of ADLER et al. [6], who found that optimisation of NIV parameters using PSG improved comfort in patients who were burdened by a severe dyspnoea occurring immediately after NIV interruption ("deventilation dyspnoea"), without compromising the quality of nocturnal ventilation (in terms of gas exchange).

Finally, it should be noted that although the evaluation of residual events under NIV in a very controlled environment using attended PSG is accurate, it cannot provide insight into the consistency over time of the residual events. Several nights of titration may be necessary to fully optimise the settings. Furthermore, home monitoring is often better tolerated by patients, and sleep quality is higher in their home environment [12]. Additionally, the effectiveness of NIV over the medium and long term also needs to be evaluated, especially for patients with progressive diseases [13]. The latest generation of monitoring systems integrated within ventilators, which can include additional monitoring peripherals such as thoraco-abdominal belts and transcutaneous $\mathrm{CO}_{2}$ measurements, may provide a more precise evaluation of the quality of home ventilation and thus allow more accurate titration of NIV parameters [14]. Another advantage of these modern systems is that some of the data recorded can be automatically transmitted to the clinician, allowing rapid intervention if NIV intolerance is detected [15].

In conclusion, the study reported by HANNAN et al. [4] is important, since it has shown that titration by PSG effectively reduces patient-ventilator asynchrony and may be useful in improving treatment tolerance in patients struggling with NIV. However, it also demonstrates that its systematic use does not improve NIV benefit in the short term for all patients. Further studies are necessary to compare the short- and long-term impact of NIV titration by PSG, not with simple daytime titration, but using titration data from new-generation ventilators. We are convinced that, in the near future, artificial intelligence will be able to analyse data from sophisticated ventilator systems and that this will greatly facilitate optimisation of ventilator parameters.

Conflict of interest: J-C. Borel is employed by AGIR à dom, a non-profit home care provider, has received grants and personal fees from Philips and Resmed, and is co-inventor of a patent with Nomics SA. J. Gonzalez-Bermejo reports personal fees for consultancy and educational activities from Resmed, Philips, Breas and Lowenstein, during the conduct of the study; personal fees for consultancy and educational activities from Synapse Biomedical, outside the submitted work.

\section{References}

1 Fanfulla F, Taurino AE, Lupo ND, et al. Effect of sleep on patient/ventilator asynchrony in patients undergoing chronic non-invasive mechanical ventilation. Respir Med 2007; 101: 1702-1707.

2 Gonzalez-Bermejo J. Framework for patient-ventilator asynchrony during long term non-invasive ventilation. Thorax 2019; in press [https://doi.org/10.1136/thoraxjnl-2018-213022]. 
3 Rabec C, Gonzalez-Bermejo J, Perrin C, et al. And the doctor answers: "Dream, Dream, I Will Be the Guardian of Your Breathing...”. J Clin Sleep Med 2016; 12: 1199-1201.

4 Hannan LM, Rautela L, Berlowitz DJ, et al. Randomised controlled trial of polysomnographic titration of noninvasive ventilation. Eur Respir J 2019; 53: 1802118.

5 Patout M, Arbane G, Cuvelier A, et al. Polysomnography versus limited respiratory monitoring and nurse-led titration to optimise non-invasive ventilation set-up: a pilot randomised clinical trial. Thorax 2019; 74: 83-86.

6 Adler D, Perrig S, Takahashi $\mathrm{H}$, et al. Polysomnography in stable COPD under non-invasive ventilation to reduce patient-ventilator asynchrony and morning breathlessness. Sleep Breath 2012; 16: 1081-1090.

7 Ramsay M, Mandal S, Suh ES, et al. Parasternal electromyography to determine the relationship between patient-ventilator asynchrony and nocturnal gas exchange during home mechanical ventilation set-up. Thorax 2015; 70: 946-952.

8 Fanfulla F, Delmastro M, Berardinelli A, et al. Effects of different ventilator settings on sleep and inspiratory effort in patients with neuromuscular disease. Am I Respir Crit Care Med 2005; 172: 619-624.

9 van der Leest S, Duiverman ML. High-intensity non-invasive ventilation in stable hypercapnic COPD: evidence of efficacy and practical advice. Respirology 2019; 24: 318-328.

10 Ogna A, Nardi J, Prigent $\mathrm{H}$, et al. Prognostic value of initial assessment of residual hypoventilation using nocturnal capnography in mechanically ventilated neuromuscular patients: a 5-year follow-up study. Front Med (Lausanne) 2016; 3: 40.

11 Janssens JP, Borel JC, Pepin JL. Nocturnal monitoring of home non-invasive ventilation: the contribution of simple tools such as pulse oximetry, capnography, built-in ventilator software and autonomic markers of sleep fragmentation. Thorax 2011; 66: 438-445.

12 Bruyneel M, Sanida C, Art G, et al. Sleep efficiency during sleep studies: results of a prospective study comparing home-based and in-hospital polysomnography. J Sleep Res 2011; 20: 201-206.

13 Gonzalez J. Respiratory Support in Chronic Obstructive Pulmonary Disease (COPD) Patients (NCT03890224). Available from https://clinicaltrials.gov/ct2/show/NCT03890224?cond=NCT03890224\&rank=1 Date last accessed: March 26, 2019.

14 Borel JC, Palot A, Patout M. Technological advances in home non-invasive ventilation monitoring: reliability of data and effect on patient outcomes. Respirology 2019; in press [https://doi.org/10.1111/resp.13497].

15 Mansell SK, Cutts S, Hackney I, et al. Using domiciliary non-invasive ventilator data downloads to inform clinical decision-making to optimise ventilation delivery and patient compliance. BMJ Open Respir Res 2018; 5: e000238. 